\title{
SCHWANNOMA OF THE NASAL SEPTUM: A CASE REPORT
}

\author{
Vallamreddy Venkateswarlu', Nara Janardhan², Vemuru Krishna Chaitanya ${ }^{3}$, Vissa Shanthi \\ 1 Professor and HOD, Department of ENT, Malla Reddy Medical College for Women, Hyderabad. \\ ${ }^{2}$ Professor and HOD, Department of ENT, Narayana Medical College and Hospital, Nellore. \\ ${ }^{3}$ Associate Professor, Department of ENT, Narayana Medical College and Hospital, Nellore. \\ ${ }^{4}$ Professor and Incharge, Department of Pathology, Narayana Medical College and Hospital, Nellore.
}

\begin{abstract}
BACKGROUND

Schwannoma is a benign neoplasm arising from nerve sheath of myelinated nerves. They are relatively frequent in head and neck region (25\%-45\%), but rarely involve nose and paranasal sinuses (4\%). The tumour arising from nasal septum is extremely rare and presents with non-specific symptoms. Nasal endoscopy and histopathological examination are essential for proper diagnosis. We report a rare case of benign schwannoma, which presented as trans-septal mass lesion. It was completely removed by transnasal endoscopic surgery. So when a mass is encountered in sinonasal tract, schwannoma also should be borne in mind in differential diagnosis.
\end{abstract}

\section{KEYWORDS}

Schwannoma Nasal Septum. Benign Tumours of Nose. Endoscopic Sinus Surgery.

HOW TO CITE THIS ARTICLE: Venkateswarlu V, Janardhan N, Chaitanya VK, et al. Schwannoma of the nasal septum: a case report. J. Evolution Med. Dent. Sci. 2016;5(21):1137-1139, DOI: 10.14260/jemds/2016/264

\section{INTRODUCTION}

Schwannoma is a benign, slow growing nerve sheath tumour that can arise in any peripheral nerve covered with supporting Schwann cells. Schwannomas of the head and neck are benign nerve sheath tumours, can occur in sites such as the neck, intracranial, orbit, intraparotid, parapharyngeal and infratemporal fossa.(1) Sinonasal schwannomas constitute rare entity constituting $4 \%$ of head and neck schwannomas. Nasal septal schwannoma is a very rare entity with very few cases previously reported in the literature.(2) We report a rare case of nasal septal schwannoma owing to its rarity.

\section{CASE REPORT}

A 35 years old female patient came to the outpatient department with complaints of nasal obstruction for the past 6 months, unilateral on the left side. It was associated with episodes of dull, recurrent headache and attacks of cold. There was no history of nasal allergy, nasal bleed and anosmia. On anterior rhinoscopy, nasal septum was observed to be deviated to left. Also a smooth, greyish white mass was observed in the floor of left nasal cavity with firm consistency, not bleeding on touch. Probe test was performed to observe the attachment of the mass, which could not be made out. Diagnostic endoscopy revealed nasal mass on both sides of posterior part of nasal septum extending into nasopharynx. CT scan of PNS was performed without contrast and a welldefined trans-septal soft tissue density involving the entire posterior part of nasal cavity extending into nasopharynx was observed.

Financial or Other, Competing Interest: None.

Submission 29-01-2016, Peer Review 27-02-2016,

Acceptance 02-03-2016, Published 14-03-2016.

Corresponding Author:

Dr. Vallamreddy Venkateswarlu,

Professor and HOD,

Department of ENT,

Malla Reddy Medical College for Women,

Suraram, Jeedimetla, Hyderabad-500055,

Telangana.

E-mail: vallamreddy.venkateswarlu@gmail.com

DOI: $10.14260 /$ jemds $/ 2016 / 264$
An endoscopic biopsy was performed using the punch biopsy technique, because it was a firm mass and the specimen obtained was sent for histopathological examination.

Histopathological examination of the biopsy specimen revealed to be benign schwannoma. After thorough counselling of patient for surgery, the tumour was excised by transnasal endoscopic surgery under general anaesthesia by piece-meal technique and bits of the specimen were sent for histopathological examination. Histopathological examination revealed the exact nature of the disease where it was observed that the histological specimen revealed densely cellular areas with characteristic nuclear palisading (Antoni type A) with a capsule.(3) Exact diagnosis of nasal septal schwannoma was confirmed after histopathology report. Patient was followed regularly at the end of 1,3 and 6 months after the surgery to observe for the recurrence and no recurrence of the disease was observed.

\section{DISCUSSION}

Schwannomas observed in head and neck region constitute between $25 \%$ and $45 \%$ of all schwannomas.(4) Schwannomas appear to be solitary, well-circumscribed tumours with an oval, round or fusiform shape. Of the schwannomas appearing in head and neck region, sinonasal schwannomas are very infrequent representing less than $4 \%$ of schwannomas of head and neck region. The order of involvement of paranasal sinuses in patients with schwannomas will be ethmoidal sinus being most commonly involved, followed by the maxillary sinus, nasal fossa and sphenoid sinus.(5) Localization to the nasal septum is exceedingly rare. Septal schwannomas arise from the autonomic or sensory nerves within the nasal septum.

The possible source of schwannoma of nasal septum includes sympathetic and parasympathetic nerves and also from the sensory nerve supply to the nasal septum. It was observed that schwannomas of sinonasal tract originate from branches of ophthalmic and maxillary divisions of trigeminal nerve.(6) These tumours which arise from autonomic nerves are non-capsulated, as perineural cells in the autonomic 
nerves were not observed. These tumours are often confused with mucocele, neurofibromas, olfactory neuroblastoma, inverted papilloma or pleomorphic adenoma of the septum which are non-capsulated and have more malignant potential.

Clinically, septal schwannomas present early due to the limited room for growth within the nasal cavity. Considering the clinical history of our patient, various differential diagnoses of sinonasal pathologies can be made as possible factors for the clinical presentation of mass in the nasal cavity. The presence of septal schwannoma is interesting, because it is a very rare presentation of a well-understood disease process. A wide variety of possible pathologies in unilateral obstruction illustrate the difficulties in diagnosis of nasal lesions. The location of the lesion does help us to narrow the diagnosis.

Schwannomas are usually well circumscribed masses, which tend to expand along peripheral nerves. Since they grow slowly, radiological studies show expansive soft-tissue masses with preservation of most bony margins. Although Magnetic Resonance Imaging (MRI) is superior in defining soft tissue tumours, CT offers better resolution of bony invasion. (7) These lesions can be differentiated from others by their characteristic nature of less aggressiveness to destroy the involved bone. However, as benign schwannoma can erode bone by pressure, bony erosion is not a criterion for malignancy. Excisional biopsy is typically curative for these patients and no reports of recurrence have been published to date.

The treatment for schwannoma is wide surgical excision through an approach that allows adequate exposure. Recurrence is rare after total removal. The surgical approach is determined according to the location and extent of the lesion. Various surgical techniques like lateral rhinotomy, external ethmoidectomy, midfacial degloving, Caldwell-Luc approach and medial maxillectomy can be employed for the treatment depending upon the size and extent of the tumour. Transnasal endoscopic sinus surgery is now widely accepted and commonly performed in cases requiring nose or paranasal surgery. $(8)$

There are usually no distinctive clinical features to be noted during clinical examination; hence, the diagnosis can only be made after histological examination. Histologically, schwannomas are encapsulated and contain hyalinized blood vessels. Macroscopically, the tumour is well demarcated, greyish-to-yellow in colour; fleshy and shiny on surface. Microscopically, it exhibits a biphasic histologic pattern of Antoni A and Antoni B areas. Antoni A tissue is composed of palisading spindle-shaped Schwann cells surrounding an acellular central region. This complete unit is called a Verocay body. Antoni B tissue is less cellular and forms no distinctive pattern.(9) Though this tumour is considered benign, it has a potential to become malignant. Histologically, the malignant potential of this tumour can be predicted based on the presence of an unequivocal malignant foci manifested by increased cellularity, numerous mitoses, anaplastic cells and invasiveness.

Schwannomas are not radiosensitive; complete surgical excision is the treatment of choice. Radiotherapy is used only in cases where there is an incomplete removal of the tumour or in cases where patients are not suitable for surgery because of underlying medical illness or in a case of a very extensive tumour.(10)
In conclusion, schwannomas of sinonasal tract should be borne in mind in differential diagnosis of mass lesion in the nasal cavity. Transnasal endoscopic surgery could successfully be used to achieve complete removal of tumour depending on its size and extent with good prognosis avoiding recurrence.

\section{ACKNOWLEDGEMENT}

We extend our sincere thanks to Dr. Subramanyam, Medical Director; Dr. Veera Nagi Reddy, Principal; Dr. Subbarao, Hospital Administrator and to the entire management and staff of Narayana Medical College and Hospital, Nellore, for their immense cooperation and support during our entire scientific work.

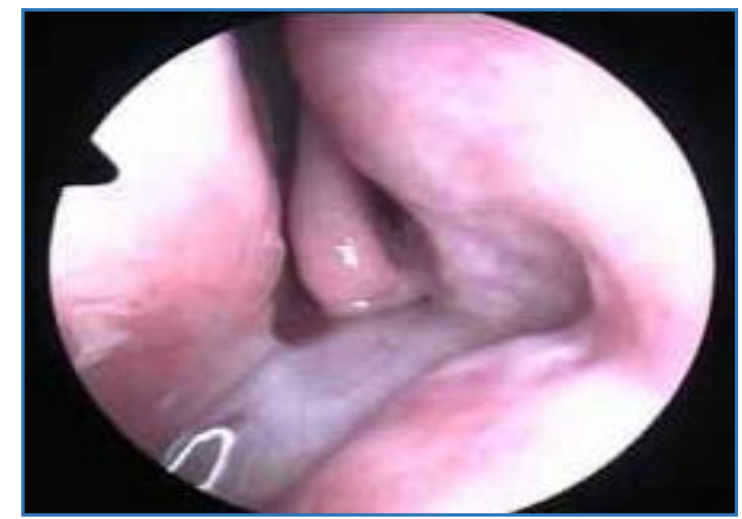

Fig. 1: Endoscopic picture of left nasal cavity showing mass in the floor extending to the nasal septum.(3)

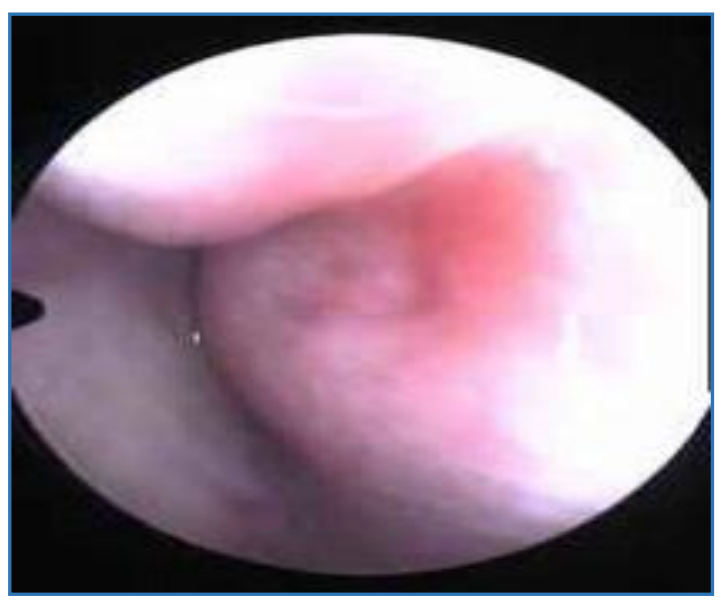

Fig. 2: Endoscopic picture of right nasal cavity showing mass arising from the nasal septum

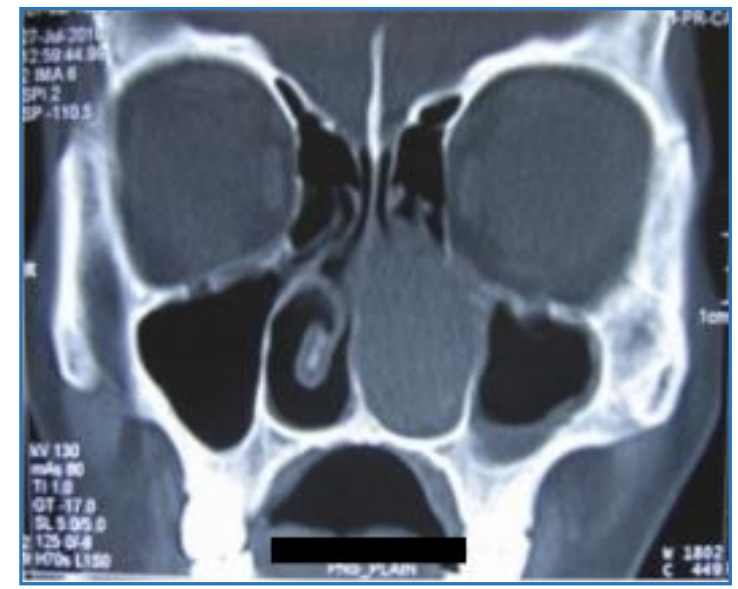

Fig. 3: CT scan (Coronal Cut) showing soft tissue density lesion in left nasal cavity 


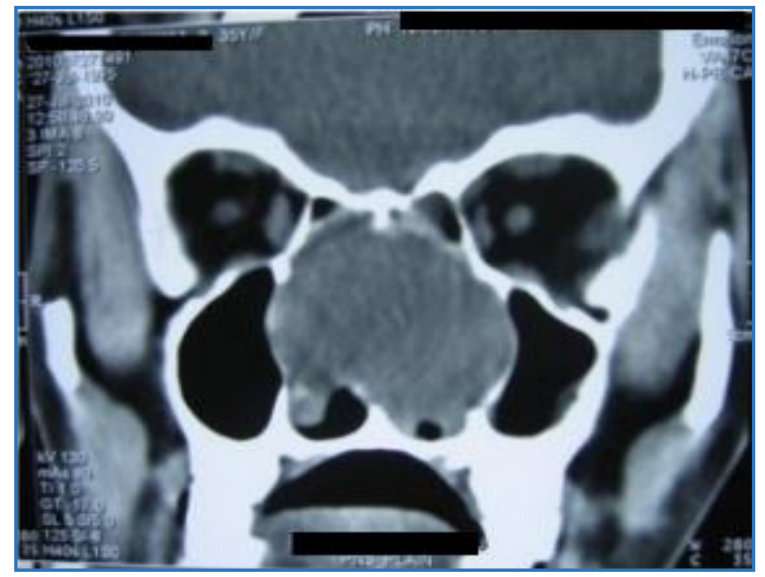

Fig. 4: CT scan showing transseptal soft tissue density lesion in the posterior cut

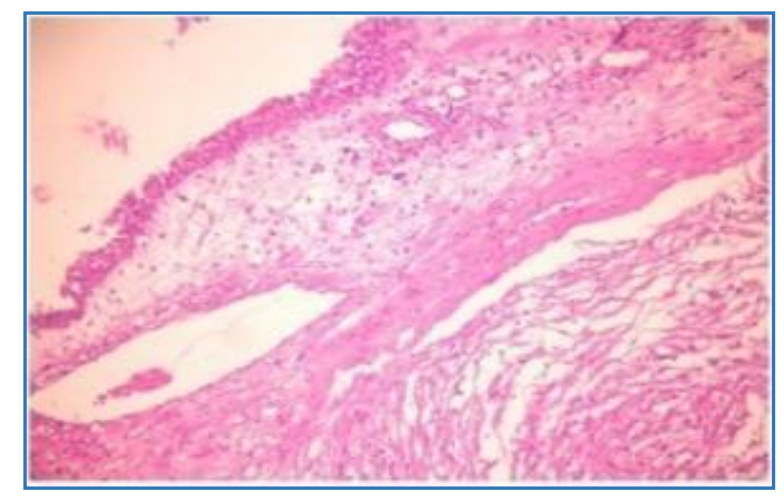

Fig. 5: Histopathological picture of the lesion showing capsule [H\&E X100]

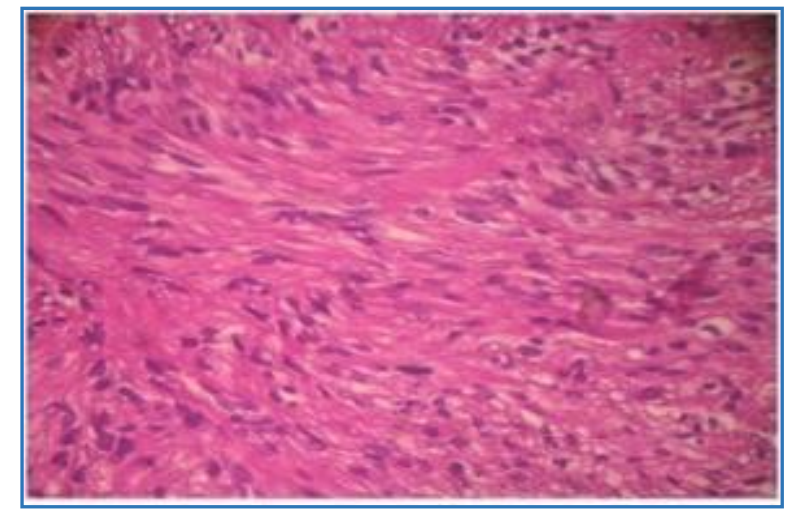

Fig. 6: Microscopic picture showing densely cellular areas with characteristic nuclear palisading (Antoni type A) [H\&E X400]

\section{REFERENCES}

1. Moukarbel Roger V, Alain N Sabri. "Current management of head and neck schwannomas." Current opinion in otolaryngology \& head and neck surgery 2005;13(2):117122.

2. Buob D, Wacrenier A, Chevalier D, et al. "Schwannoma of the sinonasal tract: a clinicopathologic and immunohistochemical study of 5 cases." Archives of pathology \& laboratory medicine 2003;127(9):1196-1199.

3. Fletcher CD, Davies SE, McKee PH. "Cellular schwannoma: a distinct pseudosarcomatous entity." Histopathology 1987;11(1):21-35.

4. Gupta TK Das, Richard D Brasfield. "Solitary malignant schwannoma." Annals of surgery 1970;171(3):419-28.

5. Loevner Laurie A, Adina I Sonners. "Imaging of neoplasms of the paranasal sinuses." Neuroimaging Clinics of North America 2004;14(4):625-646.

6. Caldemeyer KS, Mathews VP, Righi PD, et al. "Imaging features and clinical significance of perineural spread or extension of head and neck tumours." Radiographics 1998;18(1):97-110.

7. Wippold Franz J. "Head and neck imaging: the role of CT and MRI." Journal of magnetic resonance imaging 2007;25(3):453-465.

8. Pasquini E, Sciarreta V, Farneti G, et al. "Endoscopic endonasal approach for the treatment of benign schwannoma of the sinonasal tract and pterygopalatine fossa." American journal of rhinology 2002;16(2):113-118.

9. Suh JD, Ramakrishnan VR, Zhang PJ, et al. Diagnosis and endoscopic management of sinonasal schwannomas. ORL 2011;73(6):308-312.

10. Fernández PL, Cardesa A, Bombí JA, et al. Malignant sinonasal epithelioid schwannoma. Virchows Archiv A 1993;423(5):401-405. 